\title{
LOS CENTROS PÚBLICOS INTEGRADOS EN GALICIA. UN MODELO A DEBATE
}

\section{INTEGRATED PUBLIC CENTRES IN GALICIA. A MODEL TO DEBATE}

http://dx.doi.org/10.15304/ie.28.5515

\author{
María Julia Diz López \\ Grupo de investigación GEFIL \\ Departamento de Pedagoxía e Didáctica \\ Universidade de Santiago de Compostela \\ mariajulia.diz@usc.es
}

\section{RESUMEN}

El artículo se centra en la realidad de los Centros Públicos Integrados en Galicia, aunque se presentan referencias a otras comunidades autónomas. Se define qué se entiende por centro público integrado, su origen, evolución y regulación normativa. Así mismo, se exponen los principales argumentos a favor de este tipo de centros y el debate suscitado entorno a su transformación o eliminación. Finalmente se recogen unas consideraciones de la autora.

Palabras clave: centro de educación obligatoria, centro público integrado, instituto-escuela, organización escolar, sistema educativo.

\section{ABSTRACT}

This paper focuses on the reality of Public Centers Integrated in Galicia, although we present references to other autonomous communities. We wanted to define what is meant by integrated public center, its origin, evolution and normative regulation. We have also presented the main arguments in favor of those centers and the debate about their transformation or elimination. Finally, the authoress presents her own considerations about the topic.

Key words: obligatory education center, integrated public center, institute-school, school organization, educational system.

\section{INTRODUCCIÓN}

Los Centros Públicos Integrados son colegios en los que se imparte Educación secundaria obligatoria conjuntamente con la Educación primaria y/o Educación infantil ${ }^{1}$. Estos centros fueron implantados al amparo del artículo 11 de la $\operatorname{LODE}^{2}$ y de la disposición adicional sexta de la LOGSE 3 .

\section{Recibido: 8/X/2018. Aceptado: X/2018}

1 Se prevé también la impartición de otras enseñanzas de régimen general no universitario, educación de personas adultas, y/o su combinación con enseñanzas de régimen especial.

2 Ley orgánica 8/1985, de 3 de julio, reguladora del derecho a la educación.

3 Ley orgánica 1/1990, de 3 de octubre, de ordenación general del sistema educativo. 
Han pasado 19 años desde su creación en la comunidad autónoma gallega, y en la sociedad hay un debate acerca de la pertinencia de este modelo organizativo. Por ende, las últimas reformas llevadas a cabo por la Consellería de Educación han ido en la línea de suprimir algunos de dichos centros $^{4}$. Frente a ello, en las poblaciones afectadas han surgido movimientos ciudadanos que reivindican su permanencia. Pero, antes de nada, vamos a revisar el marco regulador y características de dichos centros en nuestra comunidad.

\section{MARCO NORMATIVO Y CARACTERÍSTICAS}

Los centros públicos integrados (CPI) se instauraron en Galicia en el año 1999, mediante el Decreto 7/1999, de 7 de enero, por el que se implantan y regulan los centros públicos integrados de enseñanzas no universitarias. En dicho decreto se establecieron los requisitos para su constitución y su Reglamento orgánico. La lectura del mismo nos permite apreciar que fueron concebidos para atender las necesidades educativas de zonas con características especiales, como se recoge en su disposición adicional primera:

Los actuales colegios públicos de educación primaria o de educación infantil y primaria que atiendan a poblaciones de especiales características sociodemográficas o escolares, podrán constituirse como centros públicos integrados que impartan la educación secundaria obligatoria, si así lo establece la Administración educativa.

Este decreto ha sido complementado por la Orden de 3 de octubre de $2000^{5}$, por la que se dictan instrucciones para el desarrollo del Decreto 7/1999. En ella se concretan las directrices en cuanto a la organización y funcionamiento de los citados centros. En concreto, se determinan los siguientes aspectos:

— El calendario para la elaboración, desarrollo y evaluación los documentos de organización del centro.

— Los procedimientos y plazos para que los órganos de coordinación docente realicen sus funciones.

— La organización de horarios de alumnado, profesorado y personal de servicios.

En síntesis, podemos decir que los CPI funcionan como los centros privados concertados; en el sentido de que en ellos el alumnado puede cursar toda la Educación obligatoria, e incluso en la mayoría de ellos, se imparte el segundo ciclo de Educación infantil (3-6 años). De esta manera, el

4 Por el Decreto 60/2017, de 22 de junio, de reorganización de centros docentes de determinadas localidades, se extinguió el CPI Monte Caxado (As Pontes de García Rodríguez), el CPI Plurilingüe Tomás de Lemos (Ribadavia) y el CIP da Ribeira (Porriño) se transformaron en CEIP. Mediante el Decreto 56/2018, de 9 de mayo, de reorganización de centros docentes públicos en determinadas localidades, el CPI Manuel Padín Truiteiro (Soutomaior) se transformó en un CEIP.

5 Orden de 3 de octubre de 2000 por la que se dictan instrucciones para el desarrollo del Decreto 7/1999 por el que se implantan y regulan los centros públicos integrados de enseñanzas no universitarias. 
alumnado puede ingresar en el centro con 3 años y no abandonarlo hasta los 16. Este hecho conlleva beneficios, y también, supone algunas limitaciones. No obstante, es necesario conocer la realidad de dichos centros en nuestra comunidad a día de hoy (septiembre de 2018).

\section{SITUACIÓN ACTUAL DE LOS CPI EN GALICIA}

Con la finalidad de averiguar el estado actual de implantación los CPI en Galicia, hemos consultado el buscador de centros ${ }^{6}$ de la Consellería de Educación. Según los datos disponibles, de los 306 centros de titularidad pública en los que se puede cursar Educación secundaria obligatoria, 64 de ellos son CPI. En la Figura 1 hemos representado su distribución por provincias.

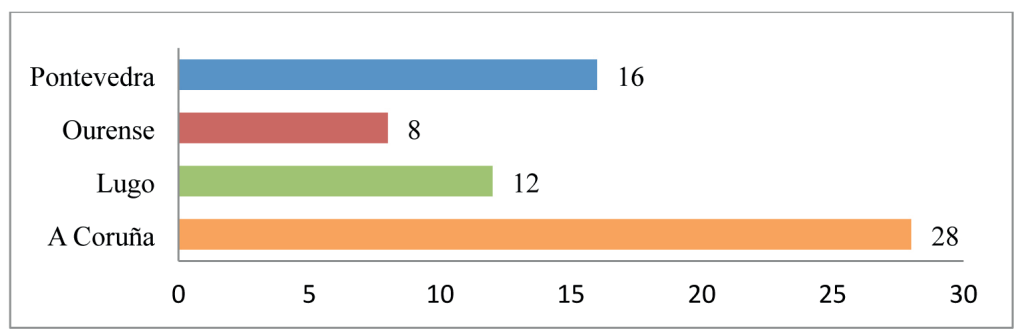

Figura 1. Distribución de CPI por provincias. Galicia 2018.

Se observa que A Coruña es la provincia gallega que cuenta con mayor número de CPI, 28 centros. En la Figura 2 ofrecemos una panorámica de su distribución geográfica.

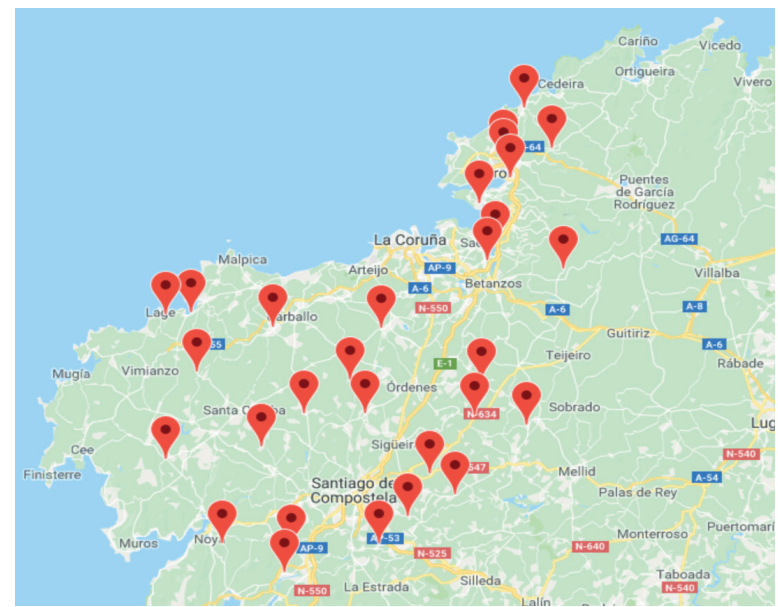

Figura 2. Distribución CPI A Coruña. Curso 18-19. Fuente: Consellería de Educación (2018) Web de centros.

6 Sitio web de la Consellería de Educación en el que se pueden realizar consultas y localizar centros educativos de Galicia: https://www.edu.xunta.es/centroseducativos/BuscaCentros.do 
Por su parte, la provincia de Lugo dispone en la actualidad de 12 CPI, lo que la sitúa como tercera en número de centros de la comunidad. En la Figura 3 se puede apreciar su distribución en el territorio.

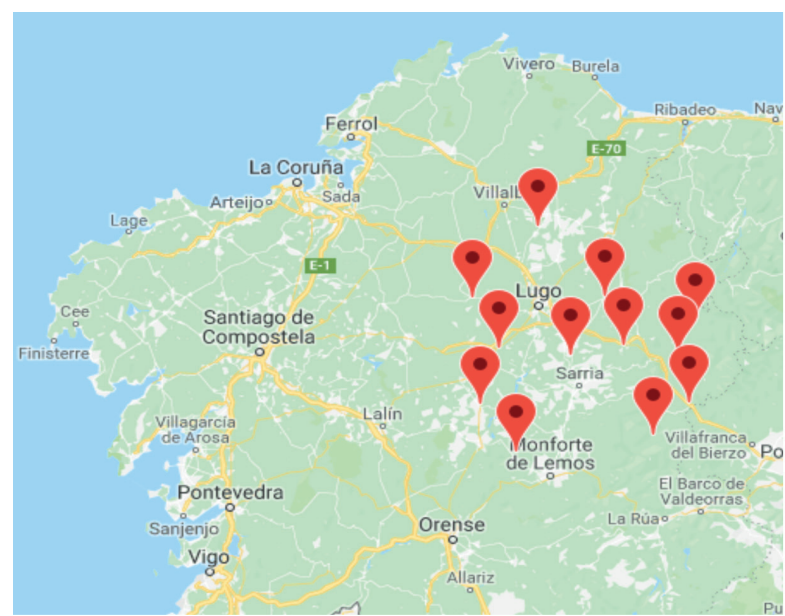

Figura 3. Distribución CPI Lugo. Curso 18-19. Fuente: Consellería de Educación (2018) Web de centros.

En cuanto a la provincia de Ourense, tenemos que indicar que en ella desarrollaban su actividad 8 CPI al comienzo del curso 2018-2019, lo cual le otorga la característica de ser la provincia gallega con menor implantación de este tipo de centros. En la Figura 4 ofrecemos su distribución.

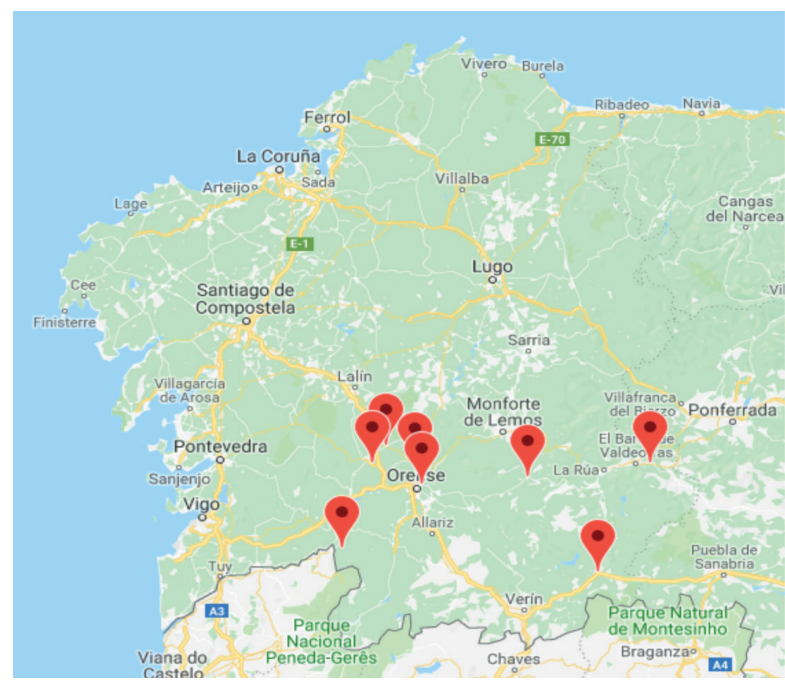

Figura 4. Distribución CPI Ourense. Curso 18-19. Fuente: Consellería de Eduación (2018) Web de centros. 
Diferente situación es la que encontramos en la provincia de Pontevedra, ya que cuenta con $16 \mathrm{CPI}$, lo que la posiciona en segundo lugar para la realidad gallega. El mapa de su distribución geográfica se recoge en la Figura 5.

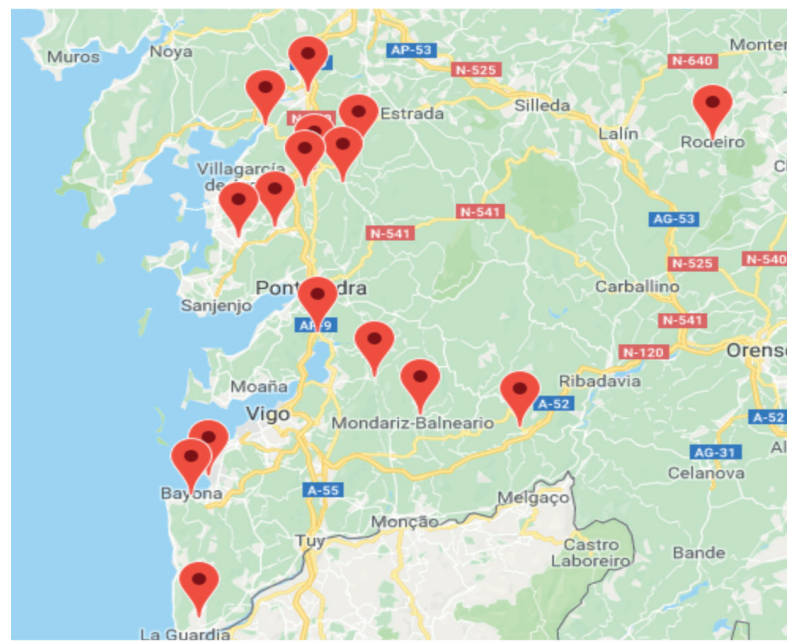

Figura 5. Distribución CPI Pontevedra. Curso 18-19. Fuente: Consellería de Eduación (2018) Web de centros

Una vez expuesto el estado actual de los CPI en Galicia conviene aproximarse al debate sobre este modelo.

\section{EL DEBATE SOBRE EL MODELO}

Los CPI se asientan en un modelo organizativo que rompe con la tradición de segregar al alumnado y al profesorado por etapas educativas en la educación pública (Rubia, 2011). Esto no es lo habitual en centros privados; de hecho, los centros privados y concertados suelen impartir toda la educación obligatoria. De esta forma, los CPI constituyen una excepción dentro de la norma.

En principio, los CPI nacen para dar solución a la separación entre la Educación secundaria obligatoria y la Educación primaria, derivada de la implantación de la LOGSE, que en la realidad gallega de los años 90 encajaba mal en el medio rural. Los IES, aún hoy en día, están ubicados en vilas y ciudades, lo cual implica el desplazamiento del alumnado del rural, para poder cursar la etapa de Educación secundaria obligatoria. Esto llevó, en 1999, al gobierno gallego a tomar la decisión de implantar este tipo de centros. Los argumentos a favor de esta modalidad organizativa se especifican en el decreto de su creación (Decreto 7/1999, de 7 de enero, p. 878):

La ampliación a diez años de la escolaridad obligatoria aconseja desarrollar formas organizativas más flexibles que las existentes, que se adapten mejor a las diversas necesidades que impone la peculiar distribución de la población de nuestra comunidad (...) para que algunos alumnos puedan realizar la obligatoriedad de la educación sin la necesidad de cambiar de centro, y un razonable aprovechamiento de los recursos existentes (...). 
En cambio, en los decretos de reorganización de centros docentes, por los que se transformaron o extinguieron CPI en los últimos dos años (Decreto 56/2018, de 9 de mayo y Decreto 60/2017, de 22 de junio), se presentan otro tipo de razones, que indican una posición opuesta. En primer lugar, se señala que la segregación del alumnado por etapas y centros educativos es ventajosa para el alumnado de Educación infantil y primaria, ya que permite disponer de más espacio, facilita la labor del profesorado y mejora las relaciones entre comunidades educativas:

De la reorganización que lleva a cabo este decreto derivan efectos pedagógicos positivos para el alumnado de educación infantil y primaria, toda vez que el traslado del alumnado de educación secundaria obligatoria permitirá a los centros disponer de más espacio para actividades docentes, y también facilitará que el profesorado pueda centrarse en proyectos educativos más acordes con la tipología del alumnado, suministrando una mejora de las relaciones en las respectivas comunidades educativas (Decreto 60/2017, p. 32467).

En segundo lugar, se exponen los beneficios potenciales para el alumnado de Educación secundaria. Dichos alumnos y alumnas gozarán de una mayor oferta educativa, de la implementación de refuerzo educativo y la adaptación al centro de cara al bachillerato:

(...) el alumnado que va a estudiar la educación secundaria obligatoria a un instituto de educación secundaria también se verá beneficiado al poder integrarse en grupos de estudio más amplios, que permiten aumentar la oferta educativa en determinadas materias que necesitan un número mínimo de alumnos/as, al facilitar las medidas necesarias de refuerzo educativo. Finalmente, este alumnado participará de un entorno socioeducativo más afín en un centro en el que podrá cursar también el bachillerato (Decreto 60/2017, p. 32467-32468).

Por último, las medidas de reorganización y desintegración de los CPI beneficiarán a toda la comunidad, ya que

(...) de las medidas de reorganización adoptadas se derivan efectos pedagógicos positivos para el alumnado de educación infantil, primaria y secundaria obligatoria afectado y, además, contribuyen a optimizar los recursos educativos tanto en lo relativo al profesorado como a las instalaciones (Decreto 60/2017, p. 32468).

Idénticas razones se alegan en el Decreto 56/2018, de 9 de mayo, de reorganización de centros docentes públicos en determinadas localidades.

En contraste con lo anterior, están las argumentaciones de las familias afectadas. De este modo, tras el anuncio en junio de 2017 de la desaparición y transformación de CPI, el diario La Voz de Galicia, recogía la siguiente información:

La decisión de la Xunta de avisar a los colegios el último día lectivo, según su versión para no generar estrés en las aulas, cayó como un jarro de agua fría en la comunidad educativa... Para las familias, la decisión de la Xunta afecta a la organización de sus casas con cambios importantes, como nuevos horarios (Sampedro, 2017, párr. 7).

La respuesta no se dejó esperar. El 25 de junio de 2017 se llevó a cabo una protesta de familias, estudiantes y vecinos procedentes de As Pontes, Outes, Ribadavia y O Porriño ${ }^{7}$, frente a la

7 Localidades a las que pertenecían los CPI que se iban a transformar o eliminar. 
Consellería de Educación en Santiago de Compostela. Contaron con el apoyo de una caravana de 120 camiones que colapsó la ciudad. En el acto se pedía la paralización de los planes de la consellería para el cierre de los CPI de estas localidades. Según se publica en el diario El País Galicia del 29 de junio de 2017, el presidente de la Confederación de ANPAS Galegas, Fernando Lacaci, manifestó al respecto, que se deberían mantener vivos los proyectos educativos que representaban los centros afectados y permitir que las familias decidiesen el tipo de enseñanza que quieren para sus hijos/ as. Las declaraciones de Xavier Docampo, representante del ANPA del CIP Monte Caxado de As Pontes, dejan claro su posicionamiento:

Se trata del centro con mayor matrícula de As Pontes, un colegio de referencia para niños con algún tipo de dificultad, con un índice bajo de fracaso escolar, pantallas digitales en las aulas compradas por la asociación y que acoge un museo etnográfico con más de 5.000 piezas (Cit. en Apestegui, 2017, párr. 3).

Desde los profesionales del sector educativo también encontramos argumentos a favor de la organización escolar en CPI. Se afirma que repercuten positivamente en la coordinación entre profesorado de distintas etapas y en el apoyo al alumnado. Así lo expresa Carmen Guaita, maestra de primaria

Al formar parte del mismo claustro, por poca coordinación que exista, hay más que en dos centros independientes, las decisiones se toman en conjunto, se puede planificar globalmente la escolarización hasta los 16, con los recursos y apoyos necesarios, la repetición se puede programar de otra forma, y se mejora en el acompañamiento (Cit. en Marqués, 2018, párr. 6).

Los argumentos sobre la reducción del fracaso escolar son apoyados por los hallazgos científicos. En esta línea se encuentran las pesquisas de Fernández Enguita y colaboradores en una investigación sobre el fracaso y el abandono escolar. En la misma, se pone de manifiesto que los momentos de cambio de etapa resultan críticos para el alumnado. El problema se acrecienta si el cambio de etapa implica cambio de centro. Según dichos autores, el momento que repercute en mayor medida en el fracaso escolar y posterior abandono, es el cambio de primaria a secundaria (Mena, Fernández Enguita y Riviére, 2010; Fernández Enguita, Mena y Riviére, 2010).

Otras investigaciones han señalado que el alumnado con más dificultades en la escuela las ve acentuadas en la transición entre etapas educativas, especialmente cuando conlleva un cambio de centro (Abrantes, 2009; Martínez Domínguez, 2011; Rossano, 2006). Las principales barreras que influyen en esta transición tienen que ver con (Antúnez, 2005; Lledó y Martínez, 2005):

- la existencia de dos culturas profesionales muy diferenciadas entre las etapas de primaria y secundaria,

- el gran número de profesorado implicado y

- la carencia de una verdadera cultura de colaboración en los centros.

Para paliar estas dificultades se recomienda "instaurar programas que atiendan al alumnado recién llegado con el objetivo de minimizar las posibles consecuencias negativas del cambio de escenario escolar" (Calvo y Manteca, 2016, p. 50). 


\section{SITUACIÓN EN OTRAS COMUNIDADES}

No podemos terminar este artículo sin aludir a la situación en otras comunidades autónomas. En Castilla y León, con un origen similar al gallego, estos centros se denominan Centros de Educación Obligatoria (CEO). Fueron creados 10 centros de estas características en el año 2002 (Decreto 34/2002 de 28 de febrero y Decreto 86/2002 de 4 de julio). En el presente curso están en funcionamiento 11 centros integrados de un total de los 234 en los que se imparte ESO (Directorio de Centros de Castilla y León, Consejería de Educación, 2018).

Por lo que atañe a Aragón, hemos de indicar que en esta comunidad se ha incorporado este modelo recientemente. En el curso 2017-18 se llevó a cabo la experiencia con 2 CPI de carácter experimental $^{8}$ y para el 2018-19 se aprobó la implantación de 12 centros integrados (Orden ECD/1082/2018, de 24 de mayo). El director general de Planificación y FP, Ricardo Almalé, manifestó en relación a los CPI que "Permiten romper la estructura tan jerarquizada de secundaria, en departamentos, y evolucionar a estructuras horizontales, a equipos de formación, de innovación, de proyectos, de orientación... Lo que facilita el tránsito" (Cit. en Marqués, 2018, párr. 14). Más aún, esta modalidad de centro permite solucionar la necesidad de plazas en secundaria derivada de los cambios demográficos. Añade que, los CPI permiten cubrir esta demanda sin ser una carga excesiva para las arcas públicas, ya que, basta con realizar pequeñas reorganizaciones administrativas y algunas transformaciones de las infraestructuras de los centros de primaria o de infantil y primaria, para convertirlos en centro público integrado.

Por lo que respecta a Cataluña, la experiencia dio comienzo a raíz de su Ley 12/2009, de 10 de julio, de Educación. En el artículo 75e se recoge la modalidad de institutos escuela y son definidos como "centros públicos que, entre otras enseñanzas de régimen general, imparten educación primaria y educación secundaria”. En el año 2012 se ha realizado la primera evaluación de este tipo de centros y en ese momento contaban con 18 institutos escuela. En el informe de la misma, elaborado por el Consell Superior d'Avaluació del Sistema educatiu (2012) se señala que este modelo está previsto para entornos complejos, especialmente desde el punto de vista sociocultural, o situaciones geográficas específicas. Tiene como finalidad potenciar la relación con las familias, especialmente en el cambio a secundaria, facilitar el conocimiento del alumnado y de su realidad social y prevenir el absentismo.

En el curso actual, están funcionando en Cataluña un total de 30 institutos escuela en los que se imparte Educación primaria y secundaria obligatoria (Generalitat de Catalunya, 2018). Existe una importante demanda de las familias y del profesorado, para el incremento de este tipo de centros, como se pone de manifiesto en el titular de prensa "Presión por el instituto escuela" publicado por elPeriódico el 16/09/2017. En el mismo se señalaba que "El PSC lleva al Parlament la petición, cada vez más extendida entre las familias, de crear más centros integrados de infantil, primaria y secundaria en Catalunya" y se añade que coincidiendo "con la presión que están ejerciendo, en cada vez más colegios, las familias para que los alumnos que terminan la primaria puedan realizar la

8 Implantación experimental de los Centros Públicos Integrados "La Jota" en Zaragoza y "Ramón y Cajal" en Ayerbe (Huesca). 
secundaria obligatoria (o al menos los primeros cursos) sin tener que cambiar de centro y siguiendo un mismo proyecto pedagógico".

\section{CONSIDERACIONES FINALES}

Los centros públicos integrados, centros de educación obligatoria o institutos escuela, según las distintas denominaciones, suponen una solución organizativa a la clásica ruptura que se produce en la educación pública entre las etapas primaria y secundaria. Encontramos antecedentes de estos centros en el Instituto Escuela creado en Madrid en 1918 por la Junta para la Ampliación de Estudios. Su proyecto pedagógico estaba inspirado en la Institución Libre de Enseñanza y en él se impartiría también formación del profesorado (Ontañón, 2007a y 2007b). Esta experiencia daría lugar a la creación de otros institutos escuela en el año 1932 en las ciudades de Barcelona, Sevilla y Valencia.

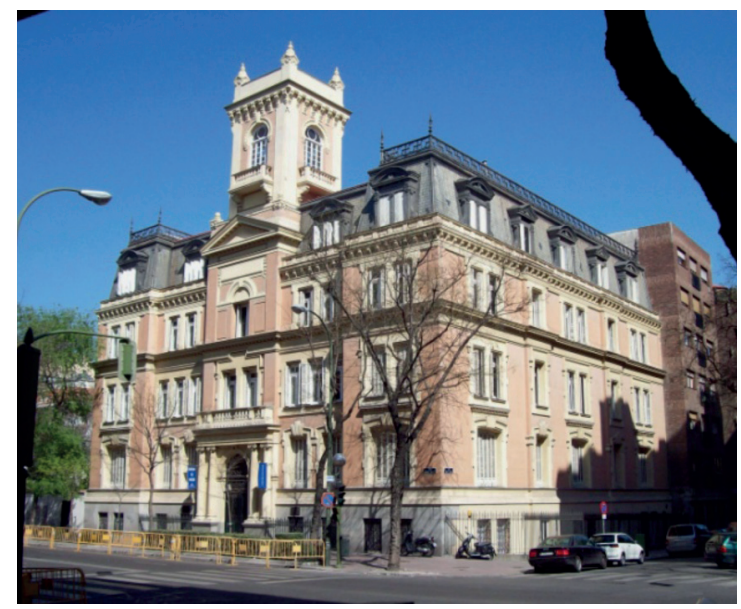

Figura 6. Instituto Internacional, primera sede del Instituto-Escuela. Fuente: García, Wikimedia Commons.

Hoy en día, la solución de un centro educativo en el que se imparta toda la formación básica constituye una alternativa habitual en la enseñanza privada y concertada y una excepción en la pública.

Este tipo de centros goza de buena reputación entre las familias y el profesorado, ya que permiten salvar las dificultades del cambio de etapa de primaria a secundaria, facilitando la transición y adaptación del alumnado. En esta misma dirección apuntan los resultados de las últimas investigaciones sobre fracaso y absentismo escolar.

La tarea de acompañar al estudiantado en la transición entre etapas se hace más sencilla si esta se produce en un mismo centro. Ello permite el trabajo conjunto del profesorado de distintas etapas, facilita su coordinación y el desarrollo de planes conjuntos de atención al alumnado y sus familias. 
No obstante, los centros integrados deben disponer de las instalaciones y espacios acordes a las necesidades del alumnado de todas las etapas, sin descuidar la infantil y primaria. Así mismo, en ellos se deben implementar todas las acciones y programas necesarios para una debida atención a la diversidad, el fomento de la convivencia y la participación de la comunidad y las familias en su proyecto educativo.

\section{REFERENCIAS}

Abrantes, P. (2009). La transición entre etapas de enseñanza en Portugal y España. Recuperado de http://www.comie.org.mx/congreso/memoriaelectronica/

Antúnez, S. (2005). El cuidado de los procesos de transición de primaria a secundaria: a modo de balance. Aula de Innovación Educativa, 142, 7-11.

Apestegui, A. (2017, junio 27). Colegios en pie de guerra contra el cierre. Radio Galicia, Cadena Ser. Recuperado de http://cadenaser.com/emisora/2017/06/27/radio_galicia/1498555718_383124.html

Aragón. Orden ECD/1082/2018, de 24 de mayo, por la que se regulan las condiciones de organización y funcionamiento de los Centros Públicos Integrados de educación básica y segundo ciclo de Educación Infantil de la Comunidad Autónoma de Aragón a partir del curso 2018/2019 (BOA de 27/06/2018).

Calvo, A. y Manteca, F. (2016). Barreras y Ayudas Percibidas por los Estudiantes en la Transición entre la Educación Primaria y Secundaria. REICE. Revista Iberoamericana sobre Calidad, Eficacia y Cambio en Educación, 14(1), 49-64. DOI: https://doi.org/10.15366/reice2016.14.1.003

Castilla y León. Decreto 34/2002, de 28 de febrero, por el que se regula la creación de los Centros de Educación Obligatoria (BOCyL de 06/03/2002).

Castilla y León. Decreto 86/2002, de 4 de julio, por el que se aprueba el Reglamento Orgánico de los Centros de Educación Obligatoria (BOCyL de 04/07/2002).

Cataluña. Ley 12/2009, de 10 de julio, de Educación (BOE de 06/08/2009).

Consejería de Educación de Castilla y León. Directorio de Centros: http://directorio.educa.jcyl.es/ es/mapa

Consell Superior d'Avaluació del Sistema educatiu (2012). Els instituts escola: aspectos organitzatius, curriculars i d'orientació. Recuperado de http://csda.gencat.cat/ca/arees_d_actuacio/ publicacions/colleccio_documents/21__ institut_escola/

Consellería de Educación (2018). Centro público integrado. Consulta realizada el 15/09/2018. Buscador de centros: https://www.edu.xunta.es/centroseducativos/BuscaCentros.do

El rechazo al cierre sorpresa de colegios decretado por la Xunta colapsa Santiago (2017, junio 29) El País Galicia. Recuperado de https:/elpais.com/ccaa/2017/06/29/galicia/1498750618_629771.html

España. Ley orgánica 8/1985, de 3 de julio, reguladora del derecho a la educación (BOE de 04/07/1985).

España. Ley orgánica $1 / 1990$, de 3 de octubre, de ordenación general del sistema educativo (BOE de 04/10/1990). 
Fernández Enguita, M., Mena Martínez, L. y Riviere Gómez, J. (2010). Fracaso y abandono escolar en España. Fundación la Caixa: Barcelona.

Galicia. Decreto 7/1999, de 7 de enero, por el que se implantan y regulan los centros públicos integrados de enseñanzas no universitarias (DOG de 26/01/1999).

Galicia. Orden de 3 de octubre de 2000 por la que se dictan instrucciones para el desarrollo del Decreto $7 / 1999$ por el que se implantan y regulan los centros públicos integrados de enseñanzas no universitarias (DOG de 02/11/2000).

Galicia. Decreto 60/2017, de 22 de junio, de reorganización de centros docentes de determinadas localidades ( $D O G$ de $04 / 07 / 2017)$.

Galicia. Decreto 56/2018, de 9 de mayo, de reorganización de centros docentes públicos en determinadas localidades (DOG de 01/06/2018).

García, L. (s. f.). Foto del Edificio del Instituto Internacional de la calle Miguel Ángel, primera sede del Instituto-Escuela [GFDL or CC BY-SA 3.0 ], from Wikimedia Commons. Descargado de https://commons.wikimedia.org/wiki/File:Instituto_Internacional_(Madrid)_01.jpg

Generalitat de Catalunya (2018). Consulta de equipaments. Recuperado de http://serveisoberts. gencat.cat/equipaments\#?titol=institut $\% 20$ escola\&categories.tema=Educaci $\% \mathrm{C} 3 \% \mathrm{~B} 3 . \% 20$ Formaci $\%$ C3\%B3\&categories.subtema=Ensenyaments $\% 20 \mathrm{de} \% 20 \mathrm{r} \% \mathrm{C} 3 \% \mathrm{~A} 8 \mathrm{gim} \% 20 \mathrm{gene}$ ral

Ibáñez, M.J. (2017, septiembre 16). Presión por el instituto escuela, El Periódico. Recuperado de https://www.elperiodico.com/es/sociedad/20170916/presion-institutos-escuela-parlamentfamilias-6289516

Lledó, A. y Martínez, C. (2005). La transición de primaria a secundaria. Un trabajo de colaboración. En VV.AA., La transición entre etapas. Reflexiones y prácticas (pp. 83-91). Barcelona: Graó.

Marqués, S. (2018, mayo 08). Centros integrados: cuando la pública imita a la concertada. Recuperado de http://eldiariodelaeducacion.com/blog/2018/05/08/centros-integrados-cuando-lapublica-imita-a-la-concertada/

Martínez Domínguez, B. (2011). Luces y sombras de las medidas de atención a la diversidad en el camino de la escuela inclusiva. Revista Interuniversitaria de Formación del Profesorado, 70(25), 165-183.

Mena, L., Fernández Enguita, M. y Riviere, J. (2010). Desenganchados de la educación: procesos, experiencias, motivaciones y estrategias del abandono y del fracaso escolar. Revista de Educación, 365 extraor., 119-145.

Ontañón, E. (2007a). El instituto-escuela, una experiencia educativa ejemplar. Circunstancia, Año V, 14. Recuperado de http://www.ortegaygasset.edu/publicaciones/circunstancia/ ano-v---n--14---septiembre-2007/ensayos/el-instituto-escuela--una-experiencia-educativaejemplar\#Resumen

Ontañón, E. (2007b, abril 23). El Instituto-Escuela un proyecto educativo vigente. El País, Recuperado de https://elpais.com/diario/2007/04/23/educacion/1177279210 850215.html

Rossano, A. (2006). El pasaje de la primaria a la secundaria como transición educativa. En F. Terigi (Comp.), Diez miradas sobre la escuela primaria (pp. 295-317). Buenos Aires: Siglo XXI.

Rubia, F. A. (2011). Los centros públicos integrados de enseñanza obligatoria: un territorio inexplorado. Fórum Aragón, 3, 33-37: 
Sampedro, D. (2017, junio 30). Las claves del conflicto con Educación. El modelo de colegio integrado gusta mucho a las familias, pero no al Estado. La Voz de Galicia. Recuperado de https:/www.lavozdegalicia.es/noticia/barbanza/outes/2017/06/30/claves-conflcto-educacion/00031498776490202714115.htm 